\title{
ESTUDIO DE LA ISLA DE CALOR DE LA CIUDAD DE IBIZA
}

\author{
Juan Antonio Serra Pardo \\ Fundación CIREM \\ (Centre d'Iniciatives i Recerques Europees a la Mediterrània) \\ Barcelona
}

\section{RESUMEN}

Los estudios del clima de las ciudades españolas se van extendiendo, teniendo cada vez más datos sobre más ciudades. Hasta el momento no parece que se haya realizado un estudio del clima urbano de la ciudad de Ibiza y mucho menos sobre el fenómeno de la isla de calor. Para remediar esto, este artículo muestra el resultado de un trabajo de investigación sobre el fenómeno de la isla de calor de la ciudad de Ibiza, donde se evidencia la existencia del fenómeno y se caracteriza. Utilizando el método de los transectos se han realizado una toma de temperaturas en dos campañas (invernal y estival). Esta toma de temperaturas ha servido para demostrar dos hipótesis de partida, por un lado que existe el fenómeno de la isla de calor en la ciudad de Ibiza y por el otro que este aparece como más intenso en verano que no en invierno debido a la actividad turística. Se ha encontrado una isla de calor con una intensidad media de $3^{\circ} \mathrm{C}$, mientras que la diferencia entre la intensidad máxima invernal y estival puede llegar a los $0,9^{\circ} \mathrm{C}$.

Palabras clave: estudio del clima urbano, isla de calor urbana, Ibiza, transectos, diferencia urbano-rural, configuración espacial.

\section{ABSTRACT}

Climate studies of Spanish cities are spreading, giving us more information about more cities. Up to the moment it does not seem that there has been realized a study of the urban climate of the city of Ibiza despite on the heat island phenomenon. To remedy this, this article shows the result of a research based on the heat island phenomenon in the city of Ibiza, demonstrating that the phenomenon appears and is characterized. Using the transects method a two campaign temperature capture has been realized (winter and summer). Temperature captures have served to demonstrate two initial hypotheses, the existence of a heat island in the city of Ibiza and that this one proves to be more intense in summer than in winter due to tourist activity. A $3^{\circ} \mathrm{C}$ average intensity heat island has been measured. While summer/winter differences come up to $0,9^{\circ} \mathrm{C}$.

Key words: urban climate, urban heat island, Ibiza, transects, urban-rural difference, spatial configuration. 


\section{Introducción}

Esta investigación pretende conseguir un mayor conocimiento de la ciudad, en este caso a partir del estudio de su clima. Este ha sido uno de los objetivos planteados en la elaboración de este trabajo. El otro gran objetivo que persigue esta investigación es el poder comprobar el cumplimiento de dos hipótesis de partida.

La primera hipótesis planteada es que en la ciudad de Ibiza se produce el fenómeno de la isla de calor. Una serie de indicios conducen a pensar en la existencia del fenómeno en esta ciudad, del que se tiene constancia, además, de que no ha sido estudiado. Estos indicios guardan relación con la estructura urbana de la ciudad, que se comentará en otro apartado.

La segunda hipótesis planteada afirma que la intensidad del fenómeno de la isla de calor en la ciudad es mayor durante el verano que no durante el invierno. La mayor actividad humana que se produce durante la época estival debido al turismo y la mayor concentración de población que éste provoca, induce a pensar que tendrá un impacto sobre el fenómeno, por lo que el comportamiento de la isla de calor urbana será diferente en invierno y en verano.

Se trata así de analizar el fenómeno de la isla de calor de la ciudad de Ibiza y sus características, con la finalidad de comprobar si las hipótesis de partida se cumplen o no.

\section{Estudio del clima urbano}

La población se muestra cada vez más sensibilizada entorno al medio ambiente y la ecología, por lo que el estudio del clima urbano se encuentra de plena actualidad, dentro de este marco.

La ciudad es la mayor construcción humana y el paisaje humanizado por excelencia, el más espectacular. Aquí es donde la acción antrópica alcanza la máxima intensidad, incluso en aspectos no visibles como la composición del aire o el clima, introduciendo una serie de impactos que tienen una gran repercusión ecológica, afectando de manera inmediata a todos los habitantes (López et al., 1993). Así las ciudades constituyen sectores singulares dentro del clima de las regiones dónde se localizan. Estas áreas urbanas aparecen como discontinuidades climáticas en estas regiones, ya que suponen una modificación de las condiciones naturales que había antes de su construcción o de las del entorno rural próximo (Moreno, 1993).

La tendencia mundial nos muestra un futuro, no muy lejano, donde la mayor parte de la población se concentrará en las ciudades, viviendo en un medio urbano. Un medio urbano que se encuentra deteriorado, donde el clima se encuentra alterado, lo cual es importante por su inmediata influencia sobre el bienestar psicofísico y en las actividades de las personas. Esto ha generado un incremento del interés del estudio e investigaciones sobre el clima urbano.

Así, a lo largo del siglo XX ha ido apareciendo una abundante bibliografía entorno al clima de las ciudades, lo cual muestra el interés de numerosos investigadores en conocer, en general, la influencia del hombre en el medio ambiente, y, en particular, las alteraciones o modificaciones climáticas antropogénicas en las ciudades.

No obstante, la percepción sensorial del clima urbano ya viene desde la antigüedad. Hay que remontarse hasta la época de griegos y romanos, quienes ya conocían que el aire de la ciudad era distinto al aire de los alrededores. Como recuerdan Landsberg, Heidorn, y otros, Séneca, en el siglo I, ya se refería al aire pesado de Roma originado por el humo de las chimeneas, de olor pestilente, y que le provocaban hasta cambios en su humor al abandonar la ciudad. 
Uno de los primeros efectos reconocidos, generados por la urbanización, fueron los cambios producidos en la composición del aire. La ciudad de Londres, a finales de la Edad Media, constituye el ejemplo más representativo de contaminación urbana, debido a esta causa. La magnitud del problema, generado por la utilización del carbón como principal combustible, era tal que en varias ocasiones, desde el siglo XII y especialmente en el siglo XVI, se tuvo que recurrir a la prohibición de la quema de carbón en la ciudad. Posteriormente, Epp en Munich, Howard en Londres o Renou en Paris realizarán estudios sobre las alteraciones del clima de las ciudades en los siglos XVIII y XIX. Los estudios urbanos se mejorarán gracias a que en los principios del siglo XIX se comenzará a emplear datos meteorológicos para analizar las alteraciones climáticas urbanas.

El empleo de datos de observatorios únicamente permite el conocer las diferencias entre puntos muy concretos, que se extrapolan al conjunto. Así, un gran adelanto vendrá de la mano del nuevo método iniciado en 1927 por Schmidt en Viena y también por Peppler en Karlsruhe, utilizando vehículos a motor con aparatos de medida para obtener numerosos datos mediante itinerarios por toda la ciudad, que será el método más indicado para conocer las diferencias dentro de la ciudad (Moreno, 1993).

Después del episodio de la II Guerra Mundial, se produce un crecimiento de las áreas metropolitanas y urbanas, donde el creciente proceso de industrialización, hace que las investigaciones y estudios sobre el clima urbano de diversas ciudades se multipliquen extraordinariamente, sobre todo, en Europa, Estados Unidos y Japón. Por otro lado, la asunción de los problemas provocados por la contaminación del aire impulsaron de un modo notable el interés por investigar y conocer con detalle los procesos atmosféricos urbanos. Así, en 1965, aparecerá la obra clásica de Chandler sobre el clima de la ciudad de Londres, que representa la primera de detalle sobre una ciudad.

En nuestro país, la percepción del clima de las ciudades aparece ya muchas veces desde el siglo XVI, al referirse a ellas los viajeros y cronistas. Así, por ejemplo, de Madrid se mencionará la extremosidad térmica, en especial el calor del verano, o las molestias ocasionadas por las basuras callejeras. En el siglo XVIII, se comienzan a realizar algunas medidas con aparatos, aunque no será hasta el siglo XIX cuando estas medidas se realicen de modo sistemático y cuando se hagan algunas consideraciones de tipo climático (López et al., 1993).

Las investigaciones sistemáticas de los elementos del clima urbano son muy recientes en nuestro país y esencialmente por obra de los geógrafos. A principio de los años sesenta se realiza una aproximación en Madrid mediante datos referentes al observatorio del Retiro y otros de aeródromos del entorno, por parte de López Gómez, con muy pocos resultados. Ya, en la década de los ochenta, se comienza a utilizar el método de los recorridos con medidas directas para detectar la isla de calor. Primero será la ciudad de Madrid y su entorno, donde un equipo del Consejo Superior de Investigaciones Científicas (CSIC) y la Universidad Autónoma de Madrid, con la coordinación de López Gómez, lo pone en práctica por primera vez. Enseguida otro grupo de geógrafos, especialmente en Barcelona (Martín Vide y Moreno) y otras ciudades catalanas, en Zaragoza o Valencia utilizarán esta técnica novedosa en nuestro país.

El interés por el estudio del clima urbano ha ido en aumento a medida que las ciudades han ido creciendo y reuniendo más habitantes. Es importante poder conseguir el conocimiento necesario para obtener unos ambientes agradables para el bienestar de las personas que residen en las ciudades. Esto debería suponer que se tuviera en cuenta en los procesos de planificación urbanística y en la edificación. 


\section{Isla de calor}

El fenómeno de la isla de calor o isla térmica urbana es aquél consistente en la mayor calidez de las ciudades, especialmente de noche, respecto al medio rural o menos urbanizado que las rodea. El centro de las ciudades, donde las construcciones y los edificios forman un conjunto más denso y compacto, suele presentar las temperaturas más elevadas. Observando gráficamente la distribución espacial de las temperaturas mediante isotermas, se puede apreciar, en general, como las isotermas presentan una disposición concéntrica alrededor del centro urbano. Así pues, se trata de una anomalía térmica positiva en los centros de las ciudades producida por ellas mismas.

Las causas que contribuyen a que se genere el fenómeno son varias:

1) Un mayor almacenamiento del calor durante el día en la ciudad, debido a las propiedades térmicas y caloríficas de los materiales de construcción urbanos y su devolución a la atmósfera durante la noche. Durante el día estos materiales se van calentando progresivamente, si bien de un modo más lento que el propio suelo desnudo o cubierto de vegetación, y durante la noche, se va enfriando, también muy lentamente, a diferencia de los suelos rurales o con vegetación, que sufren una rápida pérdida de calor por la irradiación nocturna. Por ello, el aire urbano, en contacto con los materiales de construcción, se enfría lentamente durante la noche. Este diferente enfriamiento explica la mayor intensidad nocturna del fenómeno.

2) La producción de calor antropogénico procedente de las diferentes actividades y procesos de combustión que se llevan a cabo en las áreas urbanas e industriales (por ejemplo, de la calefacción, transporte, alumbrado, industria, etc.).

3) La disminución de la evaporación, debido a la substitución de la superficie natural originaria por un suelo pavimentado (que presenta unas características diferentes) y a la eficacia de los sistemas de drenaje urbanos (alcantarillados, etc.). Esto propicia, por una aparte que se produzca una rápida escorrentía del agua tras la precipitación, mientras que por otra parte se elimina el agua de la superficie, impidiendo la posibilidad de almacenamiento de ésta en el suelo.

4) Una menor pérdida del calor sensible, debido a la reducción de la velocidad del viento. Los edificios, con su amplia variedad de formas y estructuras, se convierten en un elemento perturbador de los flujos aéreos. La rugosidad que ofrecen las edificaciones generan una serie de alteraciones en el viento, entre las cuales destaca la reducción de su velocidad, debido a la mayor resistencia a éste que ofrece la presencia de los edificios, que actúan como parapetos.

5) Un aumento de la absorción de radiación solar, debido al efecto de captura que produce la singular geometría que presentan las calles y los edificios, que contribuye a un albedo relativamente bajo. La radiación solar incidente sufre múltiples reflexiones en las fachadas y en el suelo, quedando atrapada entre las calles.

6) Una disminución de la pérdida de calor durante la noche por irradiación, debido también a las características geométricas de calles y edificios, que reducen el factor de visión del cielo, dando una menor superficie libre de cielo a la que es devuelta, sin ningún obstáculo, la irradiación nocturna.

7) Un aumento de la radiación de onda larga que es absorbida y reemitida hacia el suelo por la contaminada atmósfera urbana. La radiación de onda larga es emitida del suelo hacia la atmósfera, ante el obstáculo que representa la presencia de una capa de contaminantes en la atmósfera urbana, es absorbida un parte de esta radicación por dicha capa, que vuelve remitir la radiación hacia el suelo. Esto impide el paso 
de la radiación de onda larga hacia niveles atmosféricos superiores y su pérdida al espacio (Moreno, 1993 y 1999).

Así, el fenómeno, parece mostrar un ritmo diario diferente, donde el valor máximo de la isla de calor aparece, generalmente, después de la puesta del sol. Esto es debido a la gran inercia calórica de la ciudad, mientras que el campo se enfría más deprisa. Cabe señalar que, en grandes urbes, la diferencia puede perdurar hasta cerca del amanecer y por tanto afectar a las temperaturas mínimas. Durante el día, las áreas rurales, que presentan una baja capacidad térmica, se calientan más deprisa y el contraste respecto a la ciudad es menor, incluso puede ser esta última, más fresca debido a la sombra proyectada en calles y patios por los edificios altos. También se ha observado un diferente ritmo estacional, donde parece existir una tendencia a que durante el invierno el fenómeno sea más intenso.

Teniendo en consideración la serie de causas explicadas que pueden dar origen al fenómeno de la isla de calor urbana, la situación ideal para que se produzca dicho fenómeno sería durante el invierno, pocas horas después de la puesta de sol, con una situación de calma anticiclónica y escasa nubosidad o bien con vientos débiles y cielos despejados o poco nubosos.

Una vez que se ha originado el fenómeno de la isla de calor, éste queda caracterizado por tres parámetros que son: su intensidad, su forma o configuración y la localización del máximo térmico.

La intensidad o magnitud del fenómeno se obtiene mediante la diferencia máxima observada, en un momento determinado, entre la temperatura de un punto del centro de la ciudad y otro de su periferia. Esta dependerá, principalmente, de factores temporales (como la hora del día y la estación del año), de factores meteorológicos (como, por ejemplo, la velocidad del viento y la nubosidad) y de factores urbanos (como el tamaño de la ciudad, la población, etc.).

La configuración espacial queda reflejada gráficamente mediante la disposición correspondiente de isotermas. La forma del fenómeno no depende tanto de las condiciones meteorológicas concretas, como de factores de tipo urbano, es decir, de las características morfológicas de la ciudad. Así se puede llegar a afirmar que existen tantas formas distintas del fenómeno como morfologías distintas de ciudades. De todos modos, hay una serie de hechos o rasgos que suelen repetirse en bastantes casos.

La localización del máximo térmico se encuentra determinada por factores urbanos, por ciertas condiciones meteorológicas, como puedan ser las eólicas, y por la estación del año. Los factores urbanos que pueden afectar a la localización del máximo térmico son los mismos que afectan a la configuración. En cuanto a las condiciones meteorológicas, según la situación sinóptica y la dirección del viento, la localización del máximo pudiera sufrir un desplazamiento a sotavento del flujo dominante.

Así, las características pueden verse modificadas, y por tanto, afectadas en cada ciudad en función de diversos tipo de factores: temporales (o que hacen referencia al momento del día y a la época del año), meteorológicos (o relativos al estado del tiempo), geográficos (o de localización de la ciudad, incluyendo su topografía), y urbanos (o relativos a las especiales características urbanas de cada ciudad). Teniendo en cuenta esto, de modo general, podemos hablar de factores naturales, que son mayoría, y factores humanos.

\section{Presentación del área de estudio}

El Mediterráneo occidental es un mar de pocas y grandes islas, al contrario que el oriental, que tiene muchas y pequeñas. En el conjunto de las islas occidentales, las Balea- 


\section{Mapa de situación}
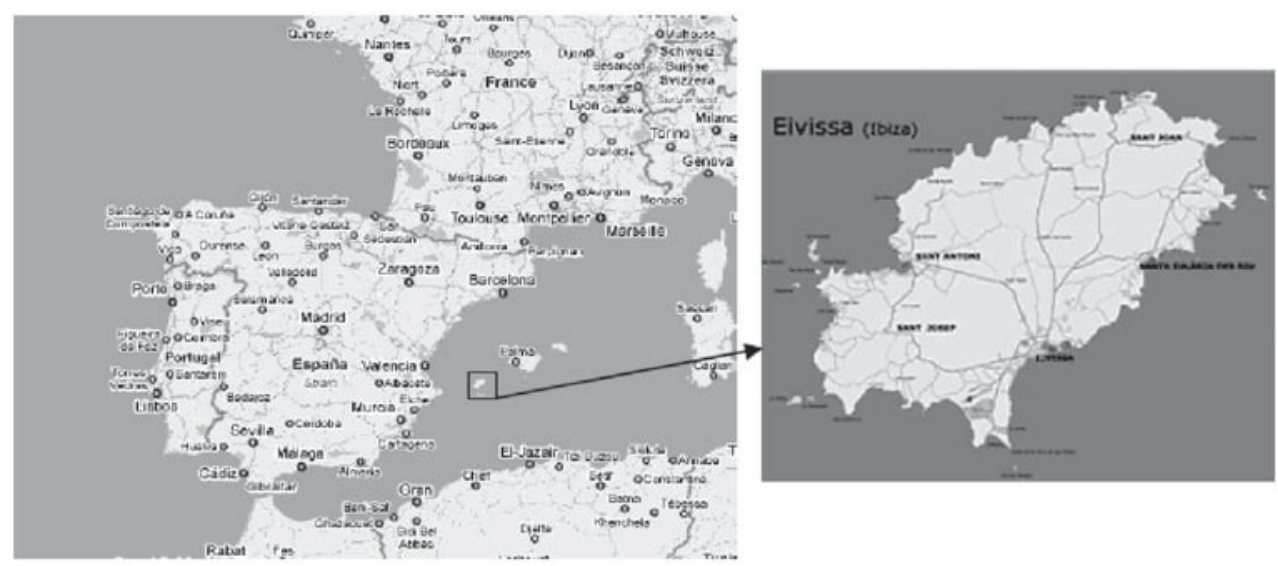

Fuente: Google Maps.

res ocupan una situación centro-oeste. Dentro del archipiélago balear, Ibiza y Formentera forman un grupo bien definido conocido por el nombre de islas Pitiusas, que recibieron de los griegos. Al sur de la isla de Mallorca y enfrente del cabo de la Nau, las Pitiusas quedan determinadas por sus coordenadas geográficas como las más occidentales y cercanas a la Península Ibérica, pero también al sector centro-occidental de la costa del norte de África. Las Pitiusas son también las que presentan una extensión más pequeña ${ }^{1}$ : Ibiza con $541 \mathrm{~km}^{2}$ y Formentera con $82 \mathrm{~km}^{2}$.

$\mathrm{Al}$ este de la isla de Ibiza se forma una extensa llanura inclinada hacia el mar, que va desde Jesús hasta ses Salines ${ }^{2}$. Esta llanura es conocida como el Pla de Vila. En los extremos, meridional y septentrional de esta llanura, alineaciones de la unidad tectónica de Ibiza, con calcáreas del jurásico y unas pocas dolomías liásicas, limitan un arco de costa que va desde Cap Martinet, al noreste, hasta la punta de ses Portes, al sur. Aquí los macizos de los montes de es Falcó y de es Corb Marí limitan ses Salines y entre ellos pasa el camino del embarcadero de sa Canal. El norte queda más accidentado, con las sierras de Balanzat, del Puig y Verda, con pequeñas elevaciones que van muriendo en el mar en la costa rocosa donde destaca el Cap Martinet, ya mencionado con anterioridad. Enfrente de éste, un pequeño macizo calcáreo avanza dentro del mar, casi como una península, cerrando una bahía de costa en formación, baja y, en un tiempo, pantanosa, donde desaguan los torrentes del Pla de Vila, los cuales contribuyen a formar el llano y la costa (Vallès, 1993).

En este llano, sobre el monte denominado Puig de Vila, se fundó la ciudad de Ibiza. A partir de este núcleo original la ciudad se fue desarrollando por las laderas de este monte de $81 \mathrm{~m}$ de altitud, hasta que alcanzó el llano continuo. Será en esta zona de llano donde se concentre el mayor crecimiento urbano de la historia de la ciudad. El fenómeno urbano se irá extendiendo por la costa próxima y hacia el interior, aprovechando las principales vías de comunicación.

1 Extensión más pequeña de las islas habitadas del Mediterráneo occidental. En este sector destaca: Sicilia con $25.708 \mathrm{~km}^{2}$, Cerdeña con $24.089 \mathrm{~km}^{2}$, Córcega con $8.628 \mathrm{~km}^{2}$, Mallorca $3.640 \mathrm{~km}^{2}$ o Menorca con 701 $\mathrm{km}^{2}$.

2 Se puede establecer una distinción entre el Pla de ses Salines y el Pla de Vila. 
FiguRa 1. Gráfico de la evolución de la población de la ciudad de Ibiza

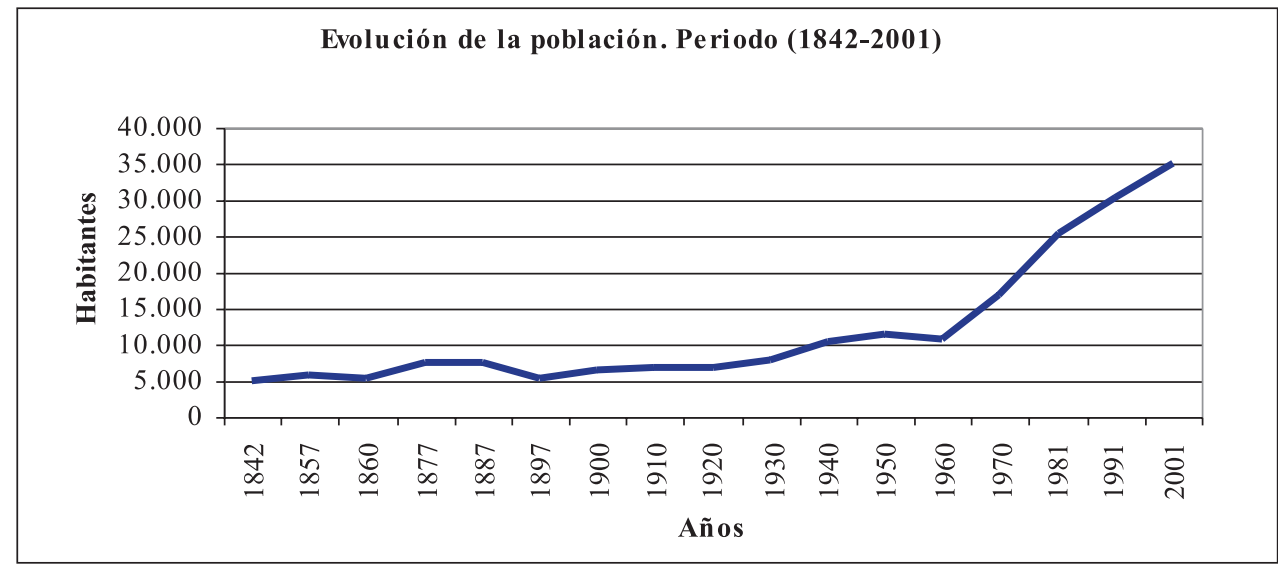

Fuente: Instituto Nacional de Estadística.

FiguRA 2. Evolución de los hogares en la ciudad para el periodo 1842-2001

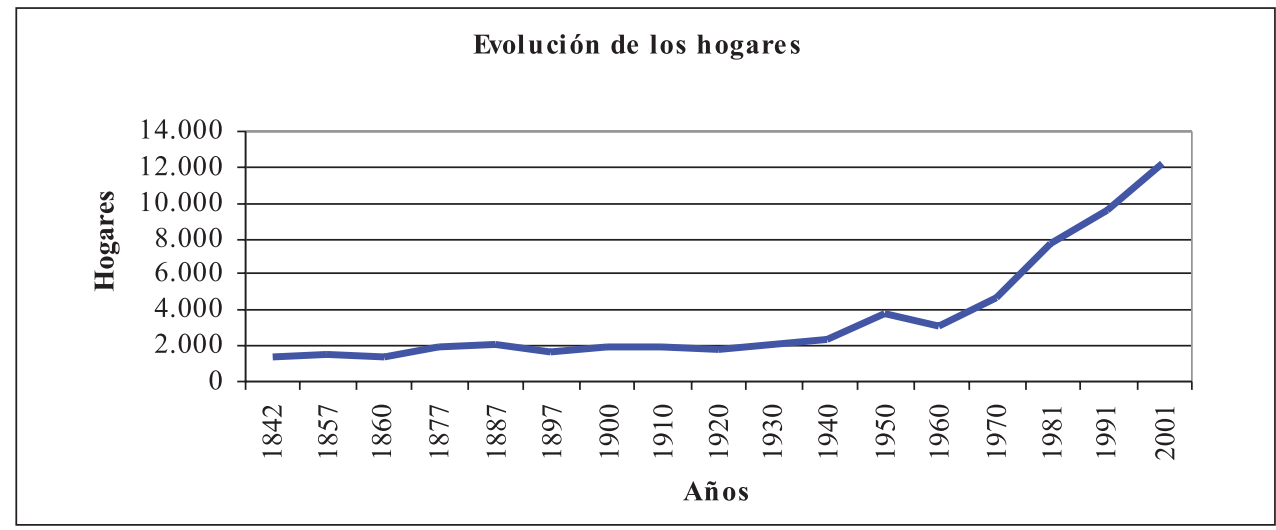

Fuente: Instituto Nacional de Estadística.

Conviene señalar cómo el turismo actuará como motor de la economía de la isla, arrastrando otras actividades. Así, la construcción, a remolque del turismo, se irá consolidando. Del mismo modo se producirá una fuerte terciarización de la economía. Todo esto trae consigo una mayor presión sobre el territorio, debido al aumento de personas, ya sean turistas o recién llegados a la isla en busca de trabajo, lo que supone un incremento de la urbanización y edificación en toda la isla, especialmente en la ciudad.

De este modo se produce un crecimiento urbano rápido y sin la adecuada planificación, lo que provocará numerosos problemas, donde la fuerte especulación tiene una gran parte de la culpa. Aunque este rápido crecimiento obedece a la imperiosa necesidad de poder dar respuesta a la demanda de vivienda provocada por la masiva llegada de contingentes de población de otros lugares. El crecimiento urbano genera un impacto sobre el medio físico, en el cual introduce modificaciones que tiene unas repercusiones. Así en esta inves- 
tigación se estudia una de las modificaciones que introduce este fenómeno urbano, como es el fenómeno de isla de calor. Una modificación que también se produce por la presión humana que supone la actividad turística.

En las figuras 1 y 2 se puede observar claramente el rápido y espectacular crecimiento de la población y del número de viviendas de la ciudad. Estos crecimientos son los que explican el aspecto actual de la ciudad, que en 40 años pasa de tener 10.753 habitantes, en 1960, a tener 34.826 habitantes en 2001. Actualmente, según el padrón municipal del 2007, la ciudad alberga una población de 44.114 habitantes.

La ciudad de Ibiza presenta una estructura urbana algo compleja. La complejidad de ésta puede venir explicada por una serie de factores:

- La reducida superficie del término municipal. Presenta una superficie total de $11 \mathrm{~km}^{2}$, lo cual lo sitúa como el segundo municipio más pequeño de las Islas Baleares.

- El gran crecimiento urbano que ha experimentado en la segunda mitad del siglo XX. Este crecimiento ha supuesto multiplicar por cuatro su superficie urbana. Crecimiento el cual, en ocasiones, no ha estado planificado de forma adecuada.

- Las diferencia topográficas entre los barrios. Unos se sitúan en terrenos llanos, mientras que otros se hayan en terrenos que presentan fuertes pendientes

- La morfología de la ciudad. Por un lado, ésta se extiende ocupando el tramo de costa existente entre Platja d'en Bossa y ses Figueretes. Por otro lado, existe una franja de unos tres kilómetros hacia el interior, por donde también se extiende la ciudad. Después aparecen unos barrios fragmentados por las vías de comunicación. También está el núcleo antiguo de la ciudad situado en un pequeño monte. Resulta así una morfología variada y compleja.

Una vez presentados algunos de los factores que explican la complejidad de la estructura urbana, conviene pasar a señalar las diferentes unidades estructurales que articulan la ciudad.

FIGURA 3. Unidades estructurales de la ciudad de Ibiza

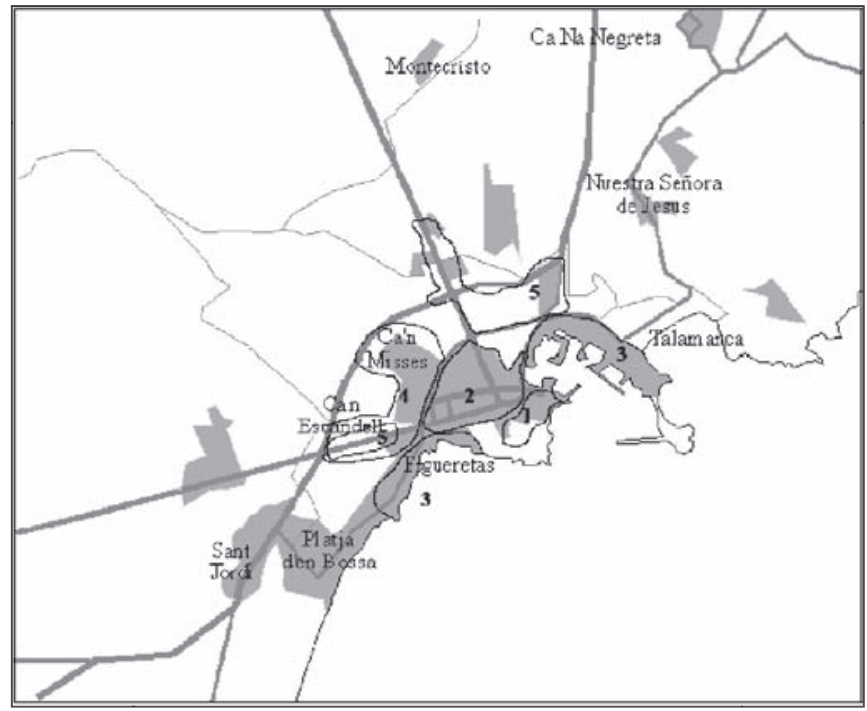

Elaboración propia. 
Como se muestra en la figura 3 y siguiendo la misma numeración se consideran un total de cinco unidades estructurales:

1. El núcleo histórico. Aquí se encuentran las barriadas de Dalt Vila, sa Penya, la Marina, y el paseo de Vara de Rey o s'Alamera. Desde el punto de vista histórico, se trata de la parte más antigua de la ciudad, donde se polarizó la vida urbana hasta los años 50, fecha a partir de la cual se comienza a expandir la ciudad. Así, este sector guarda la mayoría de los edificios históricos y de valor arquitectónico de la ciudad, siendo declarada desde 1999 Patrimonio de la Humanidad por la UNESCO. El paso del tiempo y el boom turístico han provocado la concentración de muchos establecimientos comerciales, con una clara vocación turística, con una oferta de restauración y ocio.

2. El ensanche de la ciudad de Ibiza, conocido como s'Eixample. Esta área se desarrolla a partir de los años cincuenta del siglo pasado. En esta unidad estructural se concentra al mayor número de actividades de función administrativa y comercial, además de muchas otras. Presenta unos límites septentrionales y occidentales bien definidos por el primer cinturón de la ciudad. Se trata de la unidad que se encuentra más densamente edificada y poblada de la ciudad.

3. Las zonas turísticas. Aunque se tratan de áreas distanciadas entre sí físicamente, presentan una serie de características y problemáticas comunes. Aquí encontramos los sectores de ses Figueretas, es Viver, la Platja d'en Bossa, Talamanca y el paseo Marítimo. Estas zonas turísticas de la ciudad alberga algo más de 13.000 plazas turísticas y unos 112 establecimientos hoteleros, lo que provoca que sean de las concentraciones turísticas más importantes de la isla. Conviene indicar cómo en estas zonas turísticas se va introduciendo la función residencial, especialmente en sectores como ses Figueretas y Talamanca.

4. Los barrios periféricos. En esta unidad se incluyen los barrios residenciales de Cas Serres, Ca n'Escandell y Can Misses. Aparecen como barrios de construcción reciente, sobre todo el último, con una población que mayoritariamente es inmigrante, donde dominan los procedentes de la Península Ibérica. Aunque con los cambios que se han ido produciendo en el patrón de la migración en la isla comienzan a aparecer inmigrantes de otros lugares. Es especialmente significativo cómo todavía hoy, aunque cada vez en un menor grado, aún hay actuaciones urbanizadoras pendientes, como por ejemplo, el asfaltado de calles, alumbrado público, instalaciones deportivas, etc. Por otro lado, la disponibilidad de solares vacíos en estas áreas y la buena accesibilidad desde las carreteras que conducen a la ciudad, han provocado la instalación de servicios públicos, como es el caso de los relacionados con el bienestar social en Cas Serres, o el de la sanidad e instalaciones deportivas municipales presentes en Can Misses.

5. Las zonas industriales. Quizás la que más destaca es la del barrio de Can Bofill, pero también hay otras en sa Blanca Dona, el área próxima a la estación eléctrica de GESA y alrededor de la carretera C-733. Observando su ubicación se puede apreciar cómo se encuentran alrededor de las arterias principales que comunican la ciudad con los principales núcleo urbanos de la isla. Estos sectores son donde se concentra la escasa actividad industrial de la ciudad, evidenciándose un inacabado proceso urbanizador en su interior. En algunos casos, la concentración de actividades industriales penetra en los barrios limítrofes situados en municipios cercanos (Enciclopèdia d'Eivissa i Formentera, 2001). 


\section{Metodología}

En la realización de esta investigación la técnica que se utilizó para obtener los datos fue la técnica de los transectos. Esta técnica incorpora la utilización de vehículos automóviles para realizar transectos urbanos donde se toman medidas meteorológicas localizadas en diversos puntos a lo largo de un recorrido, con lo que es posible obtener unas series de medidas meteorológicas sobre el terreno. Las medidas fueron tomadas con un termohigrómetro digital de baja inercia, que constaba con una sonda que se ubicaba en el exterior del vehículo.

La puesta en práctica de este método se realizó por primera vez en 1927 cuando Schmidt y Peppler lo aplican para el estudio del clima de las ciudades de Viena y Karlsruhe. Desde entonces se ha convertido en una técnica muy usual y representativa para el análisis de los climas urbanos (Moreno, 1993). En España, esta técnica no se utilizará hasta la década de los ochenta, utilizándose por primera vez en las ciudades de Madrid y Barcelona.

Los transectos, utilizando «estaciones meteorológicas móviles» están indicados especialmente para medir la temperatura y la humedad (Moreno, 1999). En el caso de estudio del fenómeno de la isla de calor lo más interesante es la temperatura.

La preparación y diseño de los transectos a realizar se ha de hacer tras analizar minuciosamente el plano de la ciudad en cuestión, de acuerdo con sus características urbanas, decidiendo el número de transectos y el trazado que deben seguir. En ellos se elegirán y distribuirán los puntos de observación, en los que se efectuarán las correspondientes mediciones, siendo el número preciso para tener una red de puntos suficientemente densa y dispersa por la ciudad. Conviene, además, que se seleccionen puntos que correspondan a lugares, que, a priori, es de esperar que puedan tener un comportamiento climático singular $\mathrm{y}$, por ello, ser de interés.

Así, el método de los transectos pone el acento en la obtención de una serie de medidas que van a incidir más sobre la distribución espacial y la configuración del fenómeno estudiado.

\section{Transectos}

En la realización de este trabajo de investigación, se diseñaron un total de tres transectos ${ }^{3}$ que suman una longitud de $23,3 \mathrm{~km}$, con un total de 81 puntos de medición. Además se cortan en un total de 12 puntos, para permitir un ajuste o corrección en las temperaturas anotadas y poder ser considerada como una toma de temperaturas simultánea. Estos transectos fueron numerados del 1 al 3, además se estableció un orden fijo a la hora de realizarlos, así el primero que se realizaba era el 2, después el 1 y, por último, el número 3 . Se decidió este orden por dos motivos, el primero porque el transecto 1 era el más importante para el estudio y debía ocupar un puesto intermedio que permitiera utilizarlo para comprobar la simultaneidad en la toma de temperaturas. El segundo motivo obedecía a la proximidad entre el final de un transecto y el inicio del siguiente. Era importante realizar los tres transectos en el menor tiempo posible, por lo que el tiempo dedicado del final de un transecto al inicio del otro tenia que ser el más breve posible. Por estos motivos se pensó que este era el mejor orden que se podía seguir en la realización de los transectos.

A la hora de hacer estos transectos se ha tenido en cuenta las principales calles de la ciudad, el centro urbano y los sentidos de circulación de las calles, hecho este último

3 Se pueden observar en la figura 4. 
importante, ya que ha condicionado mucho los transectos al impedir realizar algunos pensados previamente.

También se ha tenido en cuenta el poder cubrir la mayor porción de ciudad posible, así como poder registrar las temperaturas de los sectores turísticos de la ciudad, con el fin de poder realizar la comparación invierno-verano y comprobar los cambios producidos por el turismo, a fin de poder confirmar una de las hipótesis de partida.

\section{Transecto 1}

Presenta una longitud de 9,6 km y un total de 30 puntos de observación de temperatura. Es el que cubre una superficie más amplia de la ciudad, además indica la influencia de dos núcleos urbanos satélites, que han crecido bajo la influencia de la ciudad, como Sant Jordi y Jesús.

Se trata del transecto más importante, ya que transcurre por los principales sectores turísticos de la ciudad, como son Platja d'en Bossa, es Viver, ses Figueretas, el Paseo Marítimo y Talamanca. Estas áreas, sobretodo la primera, en invierno se caracterizan por los locales turísticos cerrados y por haber apenas residentes habitando. Así que servirá para establecer una comparación invierno y verano, y observar si las características de la isla del calor se ven afectadas por el turismo. También, cabe señalar que este transecto pasa por el Paseo Vara de Rey, considerado como el centro neurálgico de la ciudad, donde existe una concentración de comercios y restaurantes que en época veraniega tiene un incremento de su actividad. Otro sector inmediatamente a continuación es el puerto, donde la actividad es notablemente mayor en las noches de verano que no en las de invierno.

Este transecto es el más relevante para la observación del comportamiento diferencial de la isla de calor en verano, ya que pasa por algunas de las calles más importantes y transitadas de la ciudad. Por todos estos motivos se utilizará para ajustar las temperaturas, ya que tiene 5 puntos en común con los otros dos transectos y era el que se realizaba unas 2 ó 3 horas después de la puesta del sol.

\section{Transecto 2}

Este transecto era el que primero se realizaba de los tres. En total tiene una longitud de 7,5 km y 25 puntos de observación. Su inicio se ubica en la carretera Ibiza-San Juan a la altura del vivero de Eiviss Garden y el final se sitúa en la carretera hacia San José en el desvío hacia Sant Jordi.

El recorrido del transecto atraviesa por completo el ensanche de la ciudad de Ibiza, pasando por el sector más densamente poblado y urbanizado. También transcurre por el sector de los polígonos industriales que se ubican alrededor de la carretera de Ibiza a San Juan y por la vía que separa a los barrios periféricos de Cas Serres y Ca n'Escandell. Si el transecto 1 servía para estudiar la influencia de la mayor actividad antrópica generada por el turismo, este transecto al pasar por el sector urbano más densamente urbanizado y pasando por dos arterias importantes de la ciudad como son las calles Bartolomé Rosselló e Isidor Macabich, servirá para comprobar si se da ahí el máximo térmico.

\section{Transecto 3}

Este último transecto tiene una longitud total de 9,2 $\mathrm{km}$ y cuenta con un total de 26 puntos de observación. El inicio se sitúa en la carretera Ibiza-San Antonio enfrente del 
FiguRA 4. Mapa que muestra el recorrido de los transectos

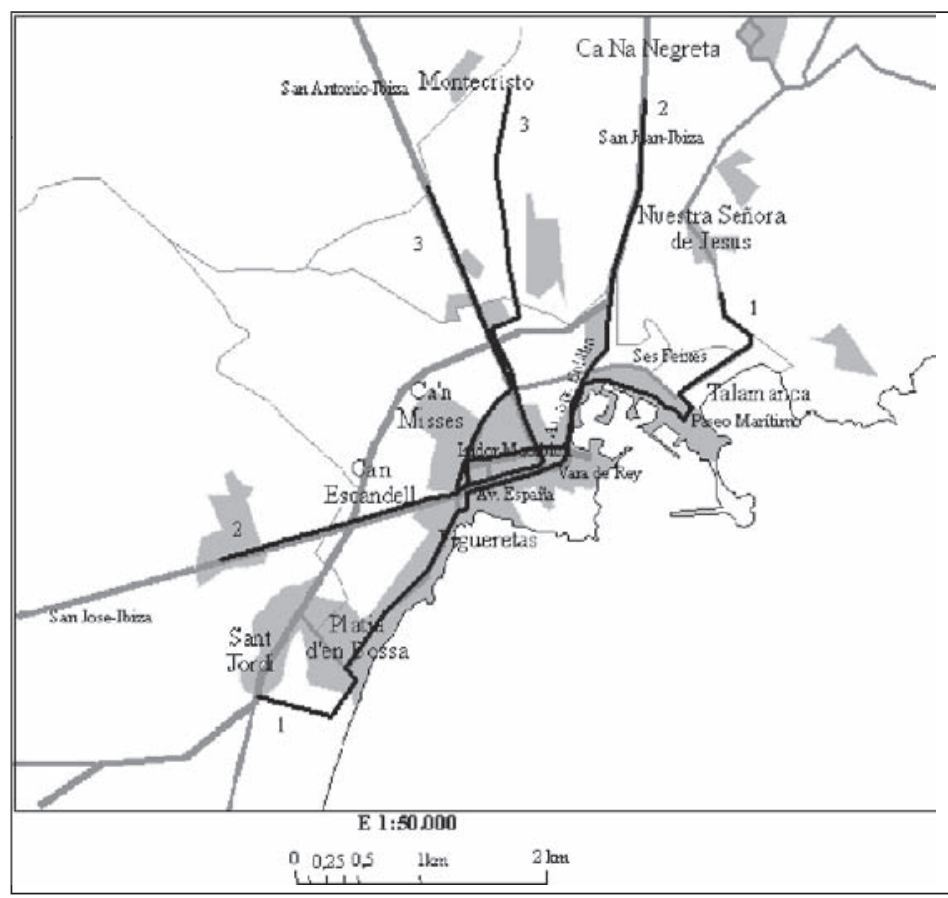

Elaboración propia.

supermercado Hipercentro, mientras que el final esta en el Cami Vell de Sant Mateu en el desvío hacía Montecristo.

El interés de este transecto reside en su paso por la zona industrial próxima a la carretera de San Antonio y por la Avenida de la Pau o primer cinturón de ronda, en el tramo donde en los últimos años se han construido nuevos edificios y donde se ubican el centro comercial de Vila Centre y los Multicines de Ibiza. También transcurre por la avenida España, en el sector de s'Eixample y por la calle Ignasi Wallis, que es otra de las principales arterias de la ciudad.

\section{Resultados}

Este trabajo de investigación se realizó mediante dos campañas de toma de temperaturas, la primera fue durante los meses de diciembre de 2005 y enero de 2006, mientras que la segunda se realizó durante los meses de julio y agosto del 2006. A la primera campaña se le denominó invernal y a la segunda, estival. En total, durante las dos campañas de tomas de temperaturas, se realizaron 31 jornadas de trabajo de campo. Esto ha supuesto realizar $722,3 \mathrm{~km}$ a través de los distintos transectos señalados. Durante las jornadas de trabajo de campo se ha realizado un registro de un total de 2.511 valores térmicos a lo largo de los 81 puntos de observación de que constaban todos los transectos. 
FiguRa 5. Intensidades medias por días

\begin{tabular}{|c|c|}
\hline Días & Intensidad \\
\hline 25 -dic & $4,0^{\circ} \mathrm{C}$ \\
\hline 26 -dic & $2,7^{\circ} \mathrm{C}$ \\
\hline 27 -dic & $1,7^{\circ} \mathrm{C}$ \\
\hline 28 -dic & $3,5^{\circ} \mathrm{C}$ \\
\hline 30 -dic & $2,6^{\circ} \mathrm{C}$ \\
\hline 31 -dic & $1,3^{\circ} \mathrm{C}$ \\
\hline 02 -ene & $1,7^{\circ} \mathrm{C}$ \\
\hline 03 -ene & $4,7^{\circ} \mathrm{C}$ \\
\hline $04-$-ene & $4,1^{\circ} \mathrm{C}$ \\
\hline 05 -ene & $5,0^{\circ} \mathrm{C}$ \\
\hline $07-$-ene & $1,6^{\circ} \mathrm{C}$ \\
\hline
\end{tabular}

Elaboración propia.

\subsection{Campaña invernal}

Durante esta campaña el transecto 1 es el que registra una mayor diferencia urbano-rural alcanzando un valor de $5,5^{\circ} \mathrm{C}$. Se trata de una diferencia muy alta, que corresponde al caso de estudio del día 3 de enero. Este mismo transecto, también es el que registra el valor más bajo de la diferencia máxima urbano-rural de entre todos los transectos. En este caso se puede apreciar que la diferencia tan sólo alcanza $1{ }^{\circ} \mathrm{C}$ durante el día 7 de enero.

En la figura 5 se muestran las intensidades medias de cada caso de estudio. Puede observarse que la mayor intensidad se registra el día 5 de enero, con un valor de $5^{\circ} \mathrm{C}$, el cual es alto. Este es el día en el que se hace la cabalgata de Reyes, lo cual significa que en las calles de la ciudad se da una importante afluencia de personas, que puede tener alguna influencia sobre la temperatura. En cuanto a la mínima intensidad, ésta se registró el día 31 de diciembre con $1,3^{\circ} \mathrm{C}$, siendo la más baja de todos los casos de estudio analizados. Fue un día donde el viento, proveniente del mar atenúo sensiblemente el fenómeno de isla de calor. La intensidad media del conjunto de casos de estudio de la campaña invernal presenta un valor de $3^{\circ} \mathrm{C}$.

En cuanto a la configuración espacial de la isla de calor convendría observar dos mapas, el primero se refiere al caso de estudio del día 5 de enero (figura 6), en el cual se registra la máxima intensidad. En este mapa se observa una isoterma cerrada a lo largo de la carretera de San José y la avenida de Sant Josep de sa Talaia. Así, el núcleo de la isla de calor se localiza en la carretera de San José, con unas temperaturas superiores a los 14 ${ }^{\circ} \mathrm{C}$. Una isoterma del mismo valor, $14^{\circ} \mathrm{C}$, también aparece a lo largo de Platja d'en Bossa. Mientras que la ciudad se encuentra bajo la influencia de isotermas de menor valor. En Sant Jordi se insinúa la presencia de una isoterma con el mismo valor que la ciudad, del mismo modo sucede en Blanca Dona y en el extremo más meridional de Puig d'en Valls. Después de este núcleo urbano, es muy llamativo la caída brusca de la temperatura, donde en poca distancia se suceden varias isotermas. 
Figura 6. Configuración de la isla de calor para el día 5 de enero

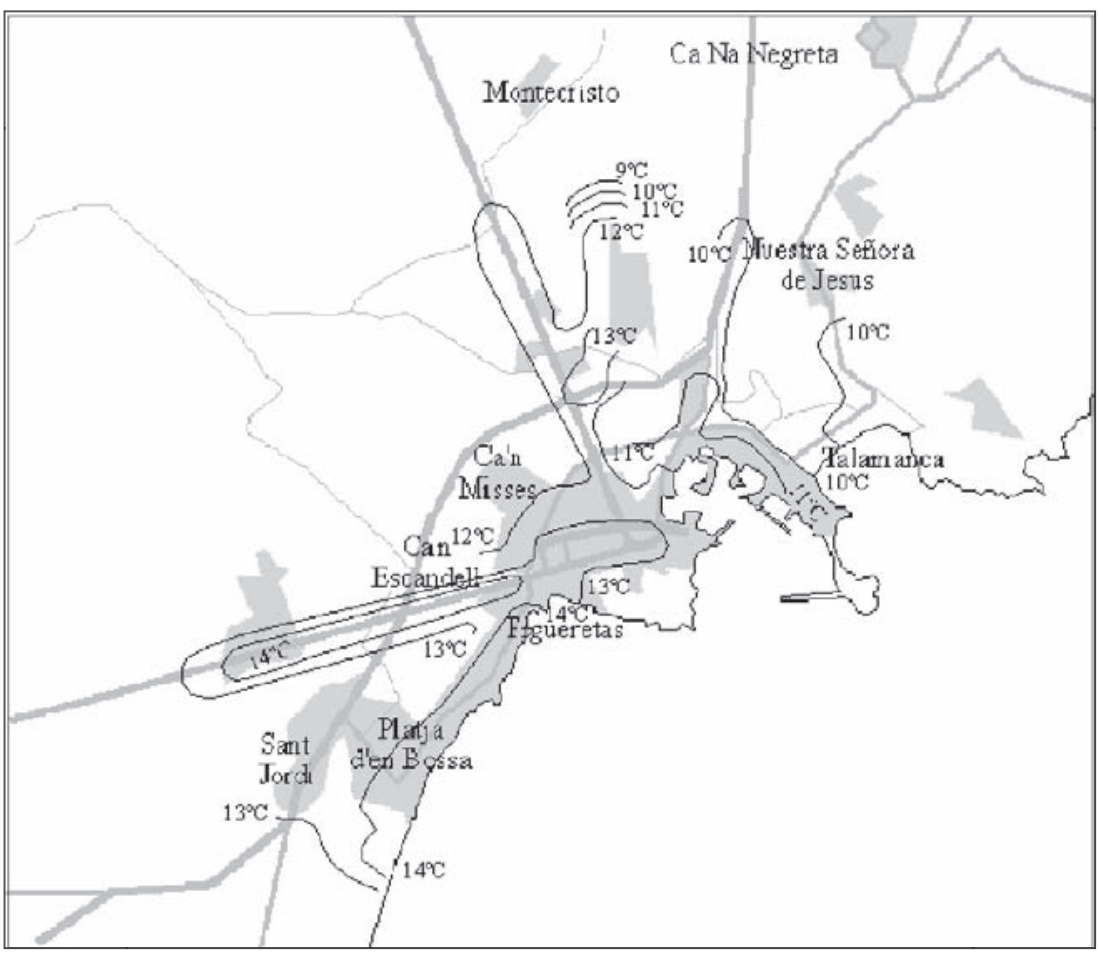

Elaboración propia.

El segundo mapa correspondería al valor medio que presentan los diferentes transectos, por lo que sería el valor medio que presenta la isla de calor durante la campaña invernal. El mapa de isotermas ${ }^{4}$ nos muestra la existencia de una isla de calor, cuya presencia viene marcada por dos isotermas concéntricas. El máximo térmico, el cual se constituye como el núcleo de la isla de calor «ibicenca», viene dibujado por la isoterma de $14^{\circ} \mathrm{C}$. Así, el núcleo de la isla de calor se encuentra situado sobre el barrio de s'Eixample, marcado por la mayor densidad edificatoria que presenta este sector, donde la avenida Isidor Macabich parece ejercer una importante influencia en la configuración del máximo térmico.

Alrededor de éste, se desarrollan las isotermas de $13^{\circ} \mathrm{C}, 12^{\circ} \mathrm{C}$ y $11^{\circ} \mathrm{C}$, lo cual señala la presencia de un significativo gradiente térmico en la ciudad, sobre todo en el primer cinturón de ronda. Las isotermas de $13^{\circ} \mathrm{C}$ y de $12^{\circ} \mathrm{C}$ se extienden a lo largo de la carretera de San José, con lo que el fenómeno de la isla de calor parece que se abarca por esta vía de comunicación. La isoterma de $12{ }^{\circ} \mathrm{C}$ se extiende también por casi toda Platja d'en Bossa y el Paseo Marítimo.

4 Mirar la figura 7. 
FIGURA 7. Configuración media de la isla de calor en invierno

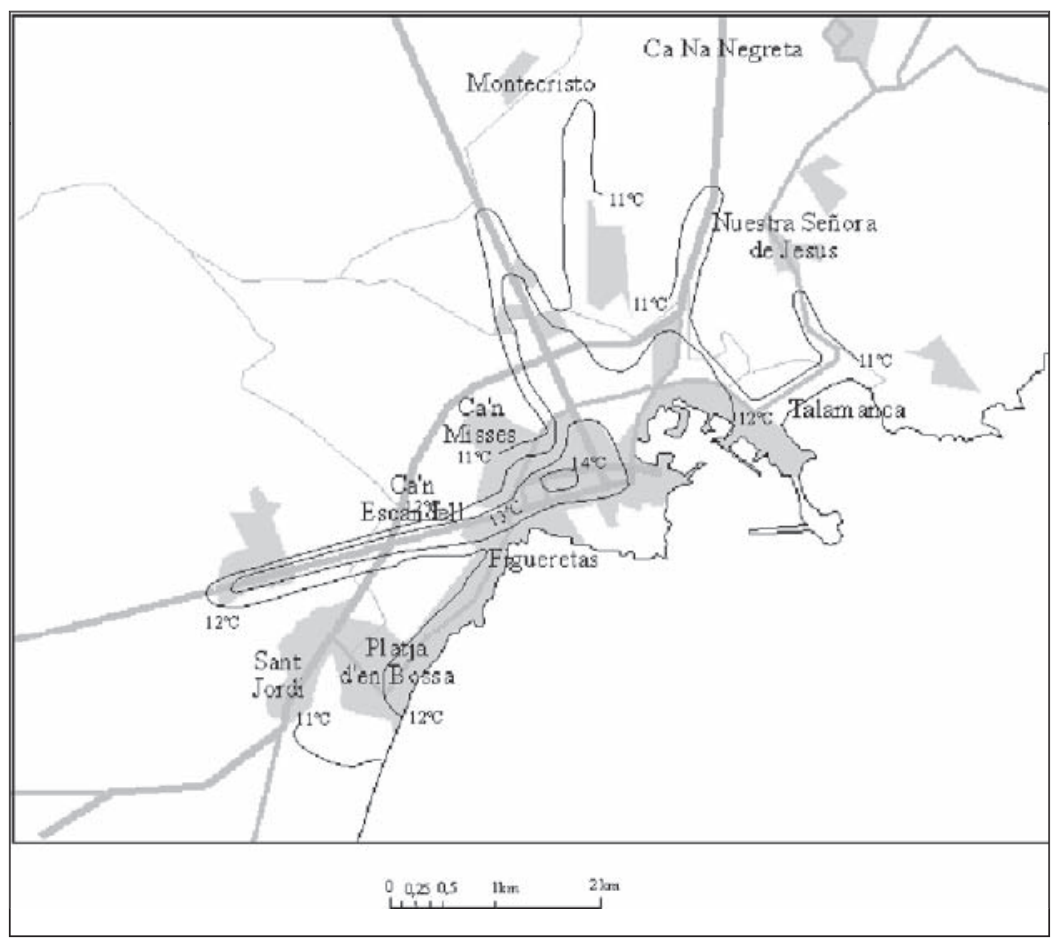

Elaboración propia.

\subsection{Campaña estival}

La máxima diferencia entre el medio urbano y el medio rural que se observa durante la campaña de verano corresponde al transecto 3 , con un valor de $6,4^{\circ} \mathrm{C}$. Este valor tan alto se registró el día 23 de julio. Mientras que el valor mínimo, para estas diferencias máximas, se observo el día 27 de julio en el transecto 1 . Este valor mínimo es de $0,9^{\circ} \mathrm{C}$. Observando la figura 8 , se aprecia cómo la mayor intensidad media se registra el día 23 de julio, cuyo valor se sitúa en $\operatorname{los} 5,4^{\circ} \mathrm{C}$. Al comparar las mayores intensidades observadas durante las campañas de invierno y de verano, esta última presenta una mayor intensidad superando en $0,4^{\circ} \mathrm{C}$ a la del invierno.

La menor intensidad que se observa durante el transcurso de esta campaña de toma de temperaturas se produce el día 27 de julio. En este día la intensidad media registrada ofrece un valor de $1,3^{\circ} \mathrm{C}$.

En cuanto a la intensidad media del conjunto de casos estudiados de la campaña estival es de $3,2^{\circ} \mathrm{C}$, superior a la intensidad de la isla de calor de la campaña invernal, en sólo $0,2^{\circ} \mathrm{C}$. Esta diferencia no representa un valor significativo, aunque si es indicativo de una, ligera, mayor intensidad de la isla de calor durante el verano. Aquí conviene recordar como el mes de agosto «atípico» ejerce una cierta influencia negativa sobre los datos obtenidos.

La lógica parece indicar que si el mes de agosto hubiera sido «normal» la diferencia en las intensidades medias (invierno-verano) sería más significativa. Si se tienen en cuenta sólo 
FIGURA 8. Intensidades medias de la isla de calor.

\begin{tabular}{|c|c|}
\hline Días & Intensidad \\
\hline 11-jul & 2,9 \\
\hline 12-jul & 3,6 \\
\hline 14-jul & 4,6 \\
\hline 16-jul & 3,4 \\
\hline 18-jul & 3,3 \\
\hline 20-jul & 4,2 \\
\hline 23-jul & 5,4 \\
\hline 24-jul & 5,2 \\
\hline 27-jul & 1,3 \\
\hline 30-jul & 2,3 \\
\hline 06-ago & 2,2 \\
\hline 10-ago & 4,0 \\
\hline 13-ago & 1,8 \\
\hline 16-ago & 1,9 \\
\hline 17-ago & 4,2 \\
\hline 20-ago & 3,5 \\
\hline 21-ago & 3,1 \\
\hline 22-ago & 4,7 \\
\hline 23-ago & 3,4 \\
\hline 28-ago & 1,5 \\
\hline
\end{tabular}

Elaboración propia.

los datos de julio, la diferencia respecto al invierno es mayor, ya que se sitúa en $0,6^{\circ} \mathrm{C}$. No obstante, para poderse establecer con mayor seguridad esta diferencia entre la intensidad invernal y estival de la isla de calor haría falta tener una mayor serie de datos.

En el mapa de isotermas correspondiente al día 23 de julio (figura 9), se puede apreciar varias isotermas cerradas presentando un valor de $28^{\circ} \mathrm{C}$. La más importante, por la superficie que abarca, se encuentra en la avenida de Sant Josep de sa Talaia, una parte de la avenida España e Isidor Macabich, estas dos situadas en s'Eixample. Un pequeño núcleo secundario se sitúa sobre la avenida de Santa Eulalia, donde en verano hay gran tránsito de personas, con destacadas retenciones del tráfico rodado, lo que contribuye a generar calor antropogénico. Otro pequeño núcleo secundario se sitúa en Platja d'en Bossa, en una zona donde también es habitual la presencia de turistas y el paso de automóviles se suele ver interrumpido por la presencia de pasos de cebra.

En el caso del mapa de isotermas, que representa la configuración media que presentaría la isla de calor de la ciudad de Ibiza en la figura 10, se puede apreciar la formación del 
Figura 9. Configuración de la isla de calor para el día 23 de julio

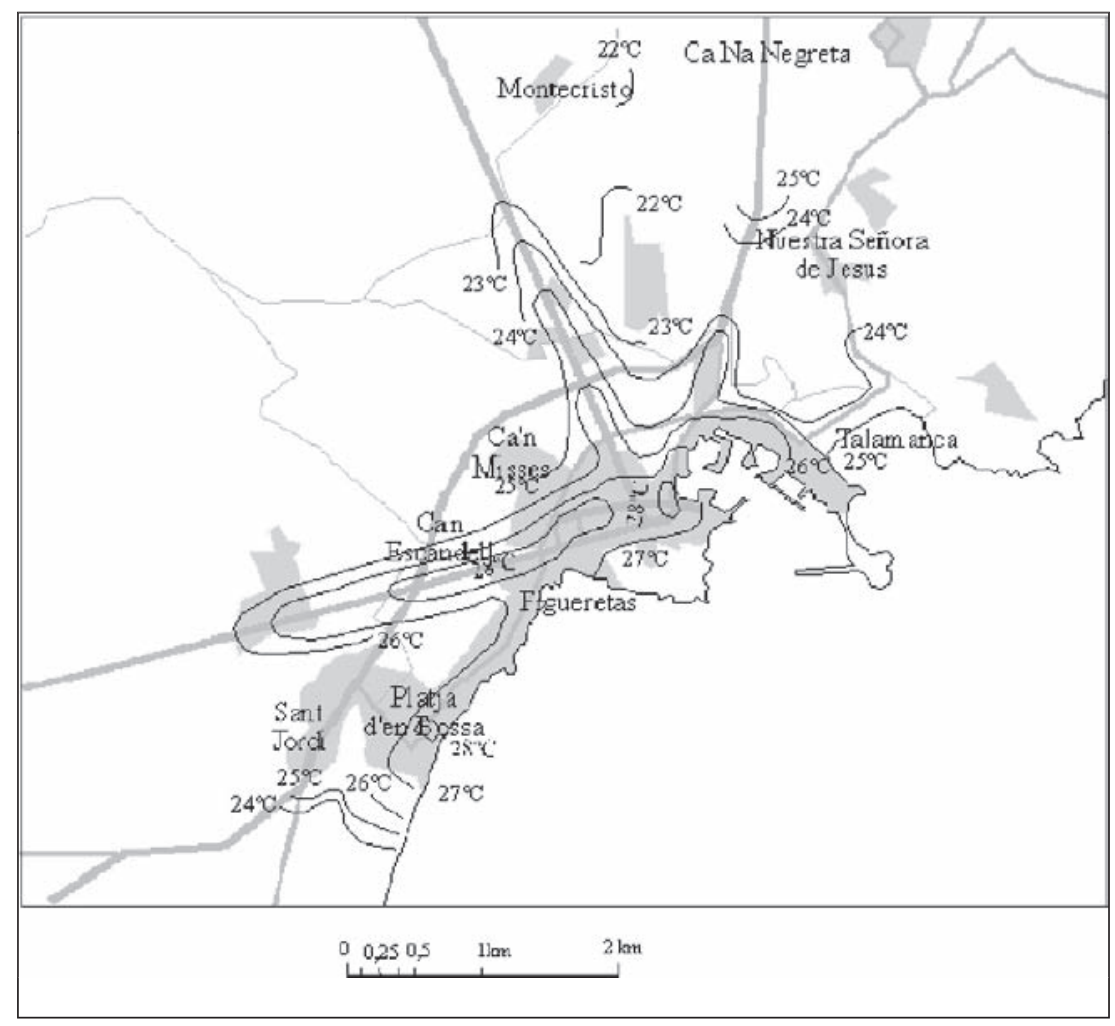

Elaboración propia.

fenómeno con la presencia de una isoterma central de $26^{\circ} \mathrm{C}$. Ésta abarca gran parte de la ciudad, justo la más densamente edificada y poblada. Aquí es donde se localiza el centro de la isla de calor, el cual vuelve a abarcar las vías urbanas más importantes de la ciudad. Este núcleo se alarga por la avenida de San Josep de sa Talaia.

Un hecho peculiar es la aparición de un centro secundario que se insinúa en los barrios de Figueretes, es Viver y Platja d'en Bossa. Estos barrios forman parte del sector turístico de la ciudad, por lo que la formación de esta isoterma es bastante reveladora sobre el impacto del turismo. Aquí se dispone una isoterma que presenta el mismo valor que el núcleo de la isla de calor.

Se puede apreciar, nuevamente, la influencia que ejercen las principales vías de comunicación que conectan la ciudad con el resto de la isla. Éstas introducen una clara modificación térmica en el medio rural.

También, una vez más, se observa la modificación térmica que generan los núcleos urbanos de Sant Jordi y Puig d'en Valls, el sector de Talamanca y la presencia de ses Feixes ${ }^{5}$.

5 Éste es un humedal próximo a la ciudad que se sitúa en la zona posterior al Paseo Marítimo. Durante el verano este sector muestra un gradiente térmico más pronunciado que no en invierno, lo que se explica, quizás, por la mayor actividad que se produce en el sector del Paseo Marítimo durante el verano. 
FIgURA 10. Configuración media de la isla de calor en verano

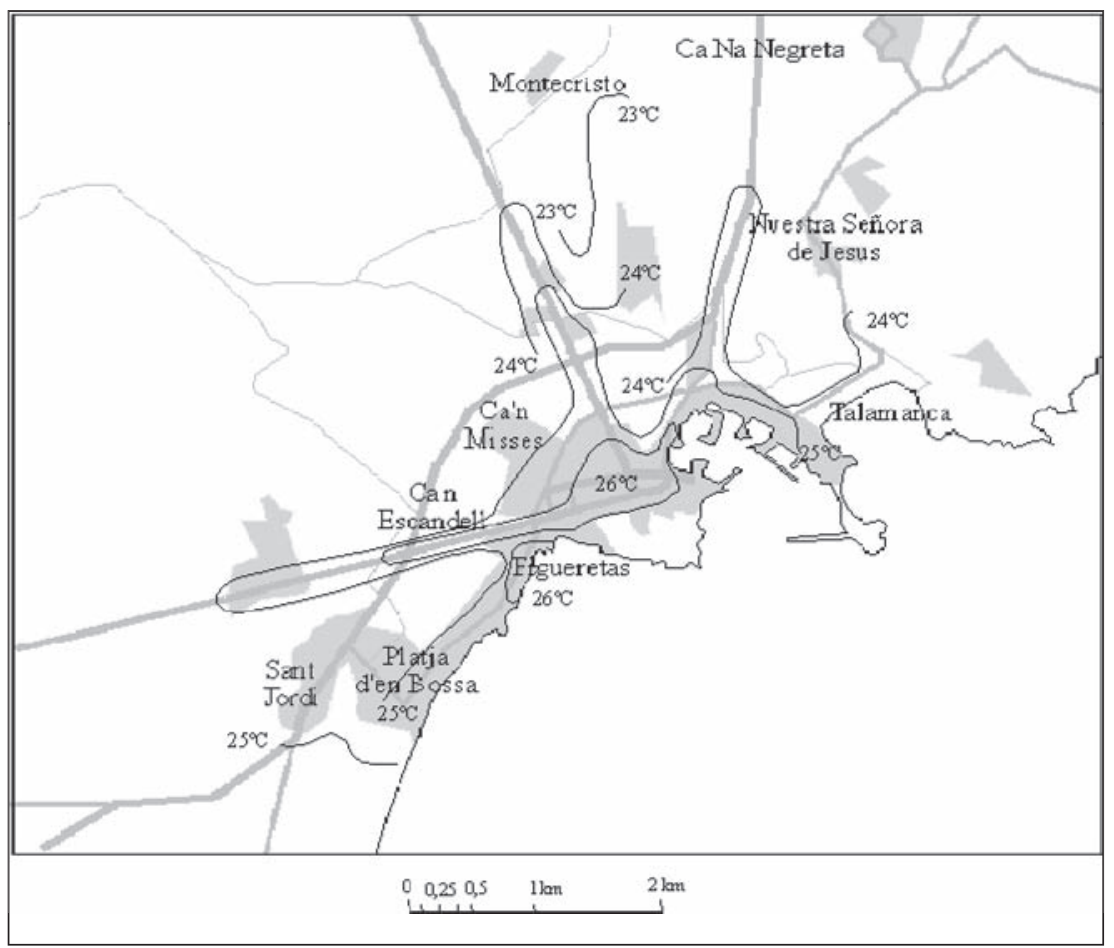

Elaboración propia.

Del mismo modo el sector de Platja d'en Bossa presenta una temperatura más elevada que el campo, desarrollada a partir de su sector más meridional.

\section{Conclusión}

Los datos recogidos durante esta investigación, aunque deberían ser mayores para establecer una caracterización más fiable de la isla de calor de la ciudad de Ibiza, son suficientes para poder afirmar que existe dicho fenómeno en la ciudad.

La intensidad del fenómeno en la ciudad de Ibiza, presenta un valor medio de $3,2^{\circ} \mathrm{C}$. No se trata de un valor muy alto, pero sí que es significativo. Las máximas intensidades parecen registrarse en verano, bajo unas condiciones meteorológicas favorables, concretamente anticiclónicas con la presencia de ola de calor. Así se ha llegado a alcanzar hasta una intensidad máxima de $6,4^{\circ} \mathrm{C}$, un valor alto. Mientras que las intensidades medias mayores se pueden llegar a situar en $\operatorname{los} 5,4^{\circ} \mathrm{C}$.

La configuración de la isla de calor muestra, en casi todos los casos estudiados, la presencia de una isoterma cerrada sobre el centro de la ciudad. Esta isoterma localiza el máximo térmico de la isla de calor. La mayor parte del barrio de s'Eixample se encuentra situado dentro de este núcleo, sobretodo en verano, lo cual es normal debido a su morfología urbana. Este máximo, en muchas ocasiones se observa cómo se puede extender hacia fuera de la ciudad a lo largo de la avenida de Sant Josep de sa Talaia. 
El núcleo de la isla de calor suele presentar una extensión más reducida durante el invierno, soliéndose ubicar en la calle Isidor Macabich. Coincide con el tramo donde los autobuses tienen sus paradas, lugar en el que el calor generado por la combustión del combustible de los vehículos es importante. En verano el núcleo presenta una mayor extensión ocupando parte de los barrios de es Pratet, la mayor parte de s'Eixample y Vara de Rey. En ocasiones, este núcleo alcanza el barrio de ses Figueretes.

Las carreteras principales que entran en la ciudad parecen desempeñar un papel de corredor, a través de las que el calor de la ciudad parece adentrarse en el campo. El caso más patente es el de la carretera de San José.

Los núcleos urbanos satélites de Sant Jordi y Puig d'en Valls muestran también la modificación que el medio urbano genera. Así, estos dos núcleos suelen mostrar $1{ }^{\circ} \mathrm{C}$ más que su entorno rural.

De este modo puede concluirse que las dos hipótesis de partida parecen cumplirse, pues por un lado queda probada la existencia del fenómeno de la isla de calor, mientras que por el otro, dicho fenómeno se muestra más intenso en verano que en invierno. Si bien la primera hipótesis se cumple de forma clara, la segunda no lo hace del mismo modo, ya que la diferencia entre las intensidades invernal y estival es de sólo $0,2^{\circ} \mathrm{C}$, aunque se observa una mayor diferencia en la intensidad máxima diaria invernal y estival.

\section{Agradecimientos}

A la Dra. M.C. Moreno García por su ayuda durante el transcurso del trabajo de investigación y en la elaboración del artículo.

\section{Bibliografía}

LÓPEZ GÓMEZ, A.; FERNÁNDEZ, F.; ARROYO, F.; MARTÍN VIDE, J.; CUADRAT, J.M. (1993): El clima de las ciudades españolas. Editorial Cátedra. Madrid, 268 pp.

MORENO GARCÍA, M.C. (1993): Estudio del clima urbano de Barcelona: la «isla de calor». Editorial Oikos-tau. Barcelona, 193 pp.

MORENO GARCÍA, M.C. (1999): Climatología Urbana. Edicions Universitat de Barcelona. Barcelona, $71 \mathrm{pp}$.

FERNÁNDEZ GARCÍA, F.; GALÁN GALLEGO, E. y CAÑADA TORRECILLA, R. (coordinadores) (1998): Clima y ambiente urbano en ciudades ibéricas e iberoamericanas. Editorial Parteluz. Madrid, $606 \mathrm{pp}$.

VALLÈS COSTA, R. (1993): Dalt Vila. Ciutat d'Eivissa. Estudi de geografia urbana. Consell Insular d'Eivissa i Formentera. Ibiza, 203 pp.

VVAA (2001): Enciclopedia d'Eivissa i Formentera, vol. 5. Universitat de les Illes Balears i Consell Insular d'Eivissa i Formentera. Ibiza, pp. 100-122. 